\title{
PRIVACY, SECURITY AND CONVENIENCE IN MOBILE DEVICE: ISSUES, DIFFICULTIES AND RECOMMENDATIONS
}

\author{
Dr. Satyendra Kurariya \\ Asstt. Professor, Department of P.G. Studies in CS \\ Mata Gujri Women's (Auto) College, Jabalpur
}

\begin{abstract}
The gap between cell phone and convenient PCs is contracting, and nearly everybody's day by day exercises are exceptionally reliant upon a gadget, known as cell phone or advanced mobile phone. So cell phone security is getting progressively significant, as business data and individual data move from the PC onto handheld storage. It is essential to do our every day exercises with no issues. Be that as it may, imagine a scenario in which four mobiles gets stolen. The paper explains issues and difficulties in security and protection of cell phones. The paper additionally suggests security highlights. Unique mark sensors and face acknowledgment are additionally featured as a developing element in security and route for handsets. In this paper I have utilized security secret key, face acknowledgment and unique mark acknowledgment to stay away from pointless informing. It works quietly out of sight and it ought to be difficult to spot.
\end{abstract}

Keywords: Smart Phone, Portable, SMS, Mobile Password, Security Secret Key, Short Message Service Center.

\section{INTRODUCTION}

The gap between cell phone and versatile PCs is contracting and is being supplanted by cutting edge cell phone known as smart phone. Smart phone offers further developed figuring capacity and availability, notwithstanding essential highlights of a cell phone. Smart phone gives computerized voice administration just as any blend of text informing, email, web browsing, still camera, MP3 player, video player, TV and organizer. Notwithstanding their implicit capacities, smart phone cell has become application conveyance stages, transforming the once resolute phones into a portable PC. At present, the mobile devices such as smart phones and tablets are not only used as a traditional mobile phone but also used as emailing, chatting, internet browsing, running a wide range of applications, file sharing, reading or editing documents, entertaining etc. From the market analysis, it was predicted that the number of usage of tablets and

\author{
Mrs. Archana Kurariya, \\ TGT, Jabalpur Vidyapeeth School \\ Jabalpur
}

smart phones would be 640 million and 1.5 billion, respectively within 2015 globally [9].

We exceptionally rely on mobile for different reasons. The unconventional reasons are:

(a) Young age utilize mobile as an absolute communication device

(b) Huge interest for portable administrations and versatile broadband access

(c) Availability of more CPU power

(d)Multitasking

(e) Multimedia informing

(f) Mobile e-business

(g)Entertainment

(h) Internet gets to

(I) Rich call

(j) Page load incredible substance and simple

route

(k) Successful publicizing (portable advertisements are unmistakably more than web based Promoting

(1) Social systems administration communications (m)Connecting with other with a style

(n) Latest and best sound, camera and video accessibility and so on. Mobile device security is

Becoming increasingly important, as business information and personal information move

From the PC to handheld storage.

In the literature study, it has been discovered that individuals are not utilizing mobile for the conventional and the previously mentioned unconventional reasons, yet they are likewise utilizing mobile for the new reasons

(a) Total human services (like symptomatic, treatment, observing, research facility administrations, release conventions, and so forth

(b) Travel manual for (discover addresses, etc) using GPS [1]. (Mir and Masood, 2002) framework

(c) Instructing.

It implies that we are utilizing mobile for all our every day exercises. Device and information both are highly sensitive and significant thus it should highly secured. Be that as it may, imagine a scenario in which our mobile gets taken. So security and protection of gadget and 
information basic issue for us. Tragically, most antitheft programming isn't free and programming which is accessible in the market sends messages to another cell phone, and furthermore at whatever point the SIM card gets changed pointlessly (like anyone can have two SIMS and they need to change SIMS as per the work necessity). In this paper to stay away from pointless informing, we have utilized security secret key, face acknowledgment and unique mark acknowledgment. It works quietly in the background and it should be difficult to spot, Even if the cheat recognizes the application, the SMS has just been conveyed.

In the event that a device is lost taken, the whole world around that individual could be undermined if those are not ensured by secret word and other client level safety efforts it is imperative to do our every day exercises with no issues.

With the enormous number of utilizations accessible for java-empowered gadgets, security is of foremost significance. Applications can deal with user sensitive information, for example, phone book or financial balance data. Additionally, Java - empowered gadgets support networking, which implies that applications can likewise make arrange associations and send or get information. Security in every one of these cases ought to be a significant concern. Malicious code has caused a great deal of mischief in the PC world, and with telephones being able to download and run applications, there is a genuine danger of confronting this equivalent danger. As of now, infections for telephone have begun have begun to rise (e.g., Cabir); various model-explicit assaults have been accounted for and mobile assaults and endeavors are beginning to get consideration in the hacker community [2]. Most of the world's smart phones now have a WiFi interface which provides another way for the user to access the Internet and associated services such as mobile operator run WiFi networks and WiFi-Calling [8].

This paper depicts the issues and difficulties in security and protection of cell phones. This paper also gives recommendations for identity privacy solutions. Unique finger impression sensors and face acknowledgment are likewise featured as a developing element in security and route for handsets. Implementations will be done with the help of mobile applications development language, J2MS [3].

\section{ISSUES AND DIFFICULTIES}

\section{(A) IMPORTANT ISSUES}

Subsequent to experiencing the writing accessible on sites, the accompanying barely any issues and difficulties in security and protection of cell phones are seen as progressively basic:
- No single security arrangement will work in the given idea of the mobile condition. Furthermore, simply existing security foundation for cell phones essentially isn't down to earth. Ventures must regard mobile security as a free assignment. What's more, as an autonomous errand, mobile utilization explicit security arrangements must be made and actualized. A thorough hazard examination of the potential security perils related with the utilization of cell phones ought to be the initial step the way of cell phone security strategy creation.

- Note that the quality of a secret password is a component of length, multifaceted nature and unpredictability. Mobile password (PIN) or security password should be solid and secure and with the end goal that no one can to identify it.

- Battery power is a basic issue. Any cell phone will be 'ON/Active ' during certain time frame depending on its utilization. Power preservation in cell phone is of vital concern. In the event that the gadget life can be delayed, the client can be increasingly beneficial and progressively happy with the utilization of this gadget.

- Security is a genuine worry for some rising applications in remote systems, while versatile protection assurance is a confounded issue, On the one hand area, following capacity gave by present day advancements makes portable clients awkward. Then again area the board for cell phones, which coordinates approaching calls and supports compulsory area administration required by governments on account of crisis, makes versatile terminals powerless against disclosure of area data. Any mobile security mechanism needs to address these apparently conflicting necessities [4].

A mobile with solid security and protection procedures should be easy to understand. This is required in light of the fact that not all the people are technically strong and have time.

\section{(B) MAJOR DIFFICULTIES}

- Cell phones are frequently excluded from the security controls routinely applied to personal computers inspired by a paranoid fear of meddling with the activities of travelling users. These gadgets are considerably more liable to be taken or appended to an unfriendly remote system than work areas that are put away safely in a corporate office behind a few layers of perimeter protection. So more grounded controls should be there in such frameworks that go on the road. It is likewise savvy to ensure that these gadgets have current programming firewalls, fix the executives, antivirus and antispyware programming. 
- Legitimately and unequivocally characterize and clarify security and protection strategy. Likewise with any security issue, the establishment of a decent reaction is strong, lear strategy that is adequately imparted to all partners. Guarantee representatives comprehend what establishes suitable and wrong utilization of big business data resources and the results of neglecting to go along.

- Know where the information lives and take reinforcement consequently inside certain time period to maintain a strategic distance from the loss of information.

- To create and keep up $100 \%$ made sure about wireless world.

- Vitality is a scant asset and a wide range of administration stop when the gadget comes up short on power.

- What to cover up and what to give regard to the security of versatile area data.

- To give area based services the alternative that full administrations will be accessible anyplace, whenever on request [5].

Security and protection arrangements should be such an extent that it is anything but difficult to utilize yet hard to guess.

\section{IDENTITY PRIVACY RECOMMENDATIONS}

The components of identity privacy solutions are summarized in Figure :

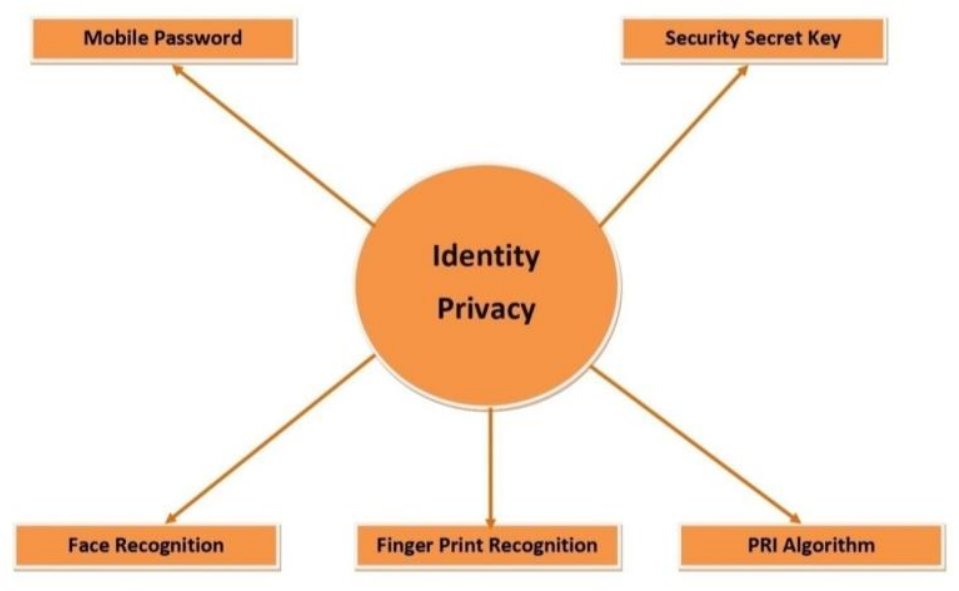

- PRI is a program for cell phones that fills in as a safe protection from being abused, misused, lost or taken. It begins naturally as the phone is turned on, and afterward consistently continues checking whether the telephone is in safe hand or SIM card has changed.
- There are two types of password and two kinds of mode. Two passwords are: mobile password and security secret key. Security secret key is required in the accompanying conditions: (a) When SIM has been changed; (b) When a unidentified client (who knows the mobile password) is attempting to utilize this telephone. Client ID should be possible consequently by two innovations: face acknowledgment; [6] and unique mark acknowledgment [7].

- Two kinds of mode are: user mode and visitor mode. In visitor mode, visitor can't get to any mobile information. He can just dial those numbers which he recalls or he can get calls. At the point when telephone is turned on, it will request for mobile password; in the event that it is alright, at that point credible client can utilize this mobile in user mode. Otherwise, it will work in visitor mode.

- Algorithm of PRI program is as follows:

$$
\text { // Mobile Switch On }
$$

Line $1: K=0$

Line 2: $\quad$ Message " Enter password"

Line 3: Read password,

Line 4:if (password=sp) then $/ *$ sp is the system password $* /$

$$
\text { Line 5: If (SIM changed) }
$$

key"

Line 6: then print "Enter security secret to Line 18.

Line 7: If (security secret key is valid) then go

Line 8: else $\mathrm{K}=\mathrm{K}+1$

Line 9: if $(K<=3)$ then go to Line 6

Line 10: else automatically send SMS containing location, person's identification

(Image, fingerprint details etc) to the previously specified number (at least two

from the recipient list), go to Line 20, endif, endif

Line 11: else

Line 12: check user identification via face recognition or fingerprint recognition

Line 13: if (user is not authentic) then

Line 14: mobile will he working in visitor

mode

Line 15: print "enter security secret key" /* if visitor want to change the mode *

Line16: if (security

secret key is not valid) then go

to Line 14. 


\section{International Journal of Engineering Applied Sciences and Technology, 2020 \\ Vol. 5, Issue 4, ISSN No. 2455-2143, Pages 272-276 \\ Published Online August 2020 in IJEAST (http://www.ijeast.com)}

18. endif

Line 17: else go to Line

Line 18: else user will be working in user mode endif, endif $\mathrm{K}=\mathrm{K}+1$

Line 19: else

Line 20: if

$(\mathrm{K}<=3)$ then go to

Line 2 else go to

Line 21 endif

$$
\text { endif }
$$

Line 21:

Turned off/Stop

The way toward sending includes the following steps:

(a) A mobile user makes a message on the user cell phone.

(b) The user mobile sends the message to a SMS server, known as Short Message Service Center (SMSC) of the necessary mobile service provider.

(c) SMSC gets the message and save a copy of this message.

(d) SMSC recognizes the location of the user mobile and sends a duplicate of the message to the user mobile. On the off chance that the user mobile isn't reachable, SMSC holds up until the user mobile is accessible.

(e) User mobile sends an acknowledgement to SMSC.

The telephone wills automatically send message to the list of beneficiaries (at any rate two) to educate that telephone is lost. Simultaneously, this message will contain the new SIM number, area and identity (picture, fingerprint etc.), the person where telephone is at present accessible. It will send three messages consistently inside a predefined time frame, so that during that length police or anyone can follow it. PRI will be in attack mode when thief changes the SIM and couldn't give security mystery key (at most multiple times); this requires driving off or rebooting the cell phone.

\section{CONCLUSION}

This paper explains the issues and difficulties in security and protection of cell phones. This paper likewise proposes calculation for security arrangements. Since information put away in the mobile is significant, so it should he exceptionally make sure about. We should not rely on device security itself. For better security, mobile manufacturer and service provider should meet up. On the off chance that these two can give the office of taking reinforcement consequently after each specific time period, at that point user information will be increasingly secure. Future work should be possible on this for battery safety.

\section{ACKNOWLEDGEMENT}

I Would like to thank all the faculty members of Computer Science Department of Mata Gujri Women's (Autonomous) College, Jabalpur for their support and guidelines. We are also thankful to the support staff and administrative staff who managed so many issues during the work.

\section{REFERENCES}

[1] Mir W. and Masood W. (2002), "GPS Technology", Proceedings of IEEE Student Conference, Vol. 2, pp. 2739 , Digital Identitier:10.1109/ISCON.2002,1214579.

Object

[2] Debbahi N., Saleh M. C and Zhioua S. (2005), "Java for mobile devices, a security study", 2 1st Annual Conference On Computer Security Applications, pp. 10 and 244, Digital Object identifier: 10.1i09/C.SAC. $200 ; .4 .2$.

[3] Jonathan K. and Sing Li (2005), Beginning J2ME: From Novice to Professiona3'-' Edition, A Press.

[4] Applewhite A (2002), "What Knows Where You Are?", PerctitPc. Computing-, IEEE, Vol. 1 , No. 4, pp. 48, Digital Object Identifier: 10.11NMPRV.'00'. 1158272.

$[5$ Campadello S.

(2004), "Peerf_toTeer_securityjn_mobile devices: a user perspe( t ive", Fourth International Conference on Peer-to-Peer Computiiv, pp. 252-257, Digital Object Identifier: 10.1109/PTP2004.13344.

[6] Voth D. (2009), "Face Recognition Technology", intelligent Systems, IEEE, Vol. 18, No. 3, pp. 4-7, Digital Object Identifier: 10.1109/N IS.2003.1200719.Reference \# 35 -20 I 1 - 12-0' LI 1.

[7] Saropourian B. (2009), "A New Approach of FingerPrint Recognition Based on Neural Network", 21'd IEEE International Conference on Computer Science and Information Technology, pp. 158-161, Digital Object Identifier: 10.1109/ICCSIT.2009.5234593.

[8] Piers O. and Borgaonkar R. (2017), Lucca Hirschi LSV, Mobile subscriber WiFi Privacy, IEEE Symposium on Security and Privacy Workshops, pp. 169-178. 
[9] Muhammad B. M , Md. Abul Kalam Azada , Athanasios Vasilakosb et al. (April 2017), Security and privacy challenges in mobile cloud computing: Survey and way ahead, Journal of Network and Computer Applications, pp. 38-54. 